\title{
INFLUENCIA DO FOTOPERÍODO EM CULTIVO DE LARVAS DE ROBALO-PEVA, CENTROPOMUS PARALLELUS
}

\author{
KENZO PEIXOTO HIRATSUKA ${ }^{1}$, VINICIUS RONZANI CERQUEIRA ${ }^{2}$ \\ ${ }^{1}$ Fundação Instituto de Pesca do Estado do Rio de Janeiro, Escritório regional da Costa Verde - Rua do Comércio, 10 - sobreloja. Centro. \\ Cep: 23900560 - Angra dos Reis, RJ - Brasil. E-mail: kenzomaricultura@yahoo.com.br \\ ${ }^{2}$ Universidade Federal de Santa Catarina, Centro de Ciências Agrárias, Departamento de Aquicultura. Rod. SC 404 Trindade \\ Cep: 88040-970 - Florianópolis, SC - Brasil - Caixa-postal: 476
}

\section{RESUMO}

Para verificar a influência do fotoperíodo em larvas de robalo-peva, conhecidas como predadoras visuais, foram realizados dois experimentos em laboratório. No primeiro, com larvas de 0 a 14 dias de idade, avaliou-se a sobrevivência e o comprimento padrão. No segundo, com larvas entre 31 a 62 dias de idade avaliou-se: sobrevivência; comprimento total; peso; índice de conversão alimentar, taxa de alimentação, taxa de crescimento específico e fator de condição de Fulton (K). As unidades experimentais eram 9 tanques (volume de 4,5 L) com 180 (experimento 1) e 35 larvas/tanque (experimento 2). Os tratamentos, em triplicata, foram 12, 18 e 24 horas de luz por dia. As larvas foram alimentadas com rotíferos (Brachionus plicatilis), no experimento 1, e matanáuplios de Artemia franciscana e ração para desmame, no experimento 2. No pimeiro experimento, os maiores valores de comprimento padrão foram nos tratamentos $24 \mathrm{~h} \mathrm{e} 18 \mathrm{~h}$. No experimento 2, o peso e o comprimento total foram maiores em $18 \mathrm{~h}$, assim como os melhores índices de conversão alimentar e taxas de alimentação. Como conclusão, a iluminação com 18 horas por dia é a mais indicada para os dois períodos larvais estudados, por favorecer o crescimento sem prejuízo para a sobrevivência.

PALAVRAS-CHAVE: fotoperíodo; robalo-peva; larvicultura; sobrevivência; crescimento

\section{ABSTRACT}

Our main objective was to verify if photoperiod has any influence on the development of fat snook (Centropomus parallelus) larvae, known as a visual feeder. Two experiments were accomplished in laboratory. In the first one, larvae were reared from eggs to 14 days after hatching. Survival and standard length were analyzed. In the second experiment, larvae were reared from 31 to 62 days and were evaluated survival, standard length, total length, weigh, feed conversion rate, feeding rate, specific growth rate and Fulton's condition factor. Nine tanks $(4.5 \mathrm{~L}$ ) were used with 180 (experiment 1 ) and 35 larvae/tank (experiment 2). The photoperiods tested were: 12, 18 and 24 hours of light/day, in triplicate. Larvae were fed rotifers (Brachionus rotundiformis) in the experiment 1 , and Artemia franciscana metanauplii and artificial weaning diet in the experiment 2 . In the first experiment, the highest values of standard length were in treatments $24 \mathrm{~h}$ and $18 \mathrm{~h}$. In the second experiment, the $18 \mathrm{~h}$ treatment had the highest values for weight, total length, feed conversion rate and feeding rate. In conclusion, we recommend the $18 \mathrm{~h}$ photoperiod treatment for both larval periods as growth was improved with no negative impact on survival.

KEY-WORDS: photoperíod; robalo-peva; larvae rearing; survival; growth

\section{INTRODUÇÃO}

Um dos principais aspectos favoráveis ao cultivo do robalo-peva é o seu valor de mercado. No Brasil, as pescarias comerciais da espécie geralmente são variáveis e baixas, ao redor de 3000 toneladas ao ano (FAO, 2006). Entretanto, os preços alcançados no mercado são considerados altos, pois exemplares da espécie são encontrados por, no mínimo, US\$ 5,00/kg (Cerqueira e Tsuzuki, 2009). Um peixe como o robalopeva poderia ser produzido em tanques-rede, reservatórios e em canais que existem nas fazendas de carcinicultura marinha (Cerqueira e Tsuzuki, 2009). Em alguns países a produção destina-se a programas de repovoamento dos estoques naturais (Brennan et al., 2006), podendo representar uma boa opção para a pesca esportiva. Entretanto, no Brasil, a espécie apresenta grande importância social, pois a maior parte de sua captura é diretamente relacionada com a pesca artesanal (Cerqueira, 2002).

Espécies do gênero Centropomus são eurihalinas, diádromas e estuarino-dependentes. $\mathrm{O}$ robalo-peva, Centropomus parallelus, é um centropomídeo americano que se distribui desde o meio da costa atlântica dos Estados Unidos até a costa da região sul do Brasil. O valor ótimo de temperatura para seu desenvolvimento se encontra entre $25{ }^{\circ} \mathrm{C}$ e $30{ }^{\circ} \mathrm{C}$, podendo tolerar temperaturas mínimas dê $10{ }^{\circ} \mathrm{C}$ (Rivas, 1986).

No cultivo de larvas de peixes marinhos pode ocorrer grandes mortalidades e baixa qualidade das mesmas, devido a condições de cultivo inadequadas, tais como a temperatura, fotoperíodo, intensidade luminosa e a cor dos tanques (Efthimiou et al.,1994). O melhor entendimento destes fatores pode melhorar significativamente o cultivo larval (Hart et al., 1996).

Geralmente longos fotoperíodos podem influenciar positivamente no desempenho de larvas de peixes, provavelmente devido à melhora na avaliação do alimento (Boeuf e Le Bail, 1999).

Para o robalo asiático Lates calcarifer, verificouse que larvas, de 2-10 dias após eclosão, cresceram progressivamente em condições de 8, 16 e 24 horas de luz por dia. A taxa de sobrevivência não diferiu 
significativamente (Barlow et al., 1995). Entretanto, investigando o efeito do fotoperíodo em crescimento e sobrevivência em larvas de robalo europeu, Dicentrarchus labrax, foram obtidos melhores resultados em sobrevivência e menor crescimento em comprimento total sob um fotoperíodo de 9 horas de luz, quando comparado com um fotoperíodo de 24 horas (Cerqueira e Chatain, 1991). Experimentos nesta área são importantes para aperfeiçoar a produção de robalopeva em laboratório. A larva desta espécie é predadora visual, necessitando de luz para sua alimentação, pois em sua ausência, pode ocorrer $100 \%$ de mortalidade (Cerqueira e Brugger 2001). Portanto, juntamente com o aumento do fotoperíodo, entende-se que seja necessário um aumento correspondente na oferta de alimento, para verificar se em fotoperíodos longos há maiores taxas de alimentação, gerando um balanço energético positivo, decorrente da maior atividade dos peixes influenciados pela luz.

O presente trabalho teve como objetivo comprovar se um incremento do fotoperíodo, com maior disponibilidade de alimento, teria influência no desenvolvimento de larvas de robalo-peva $C$. parallelus. Avaliou-se o crescimento e a sobrevivência em duas faixas de idade, o que pode contribuir para aperfeiçoar a larvicultura desta espécie em laboratório.

\section{MATERIAL E MÉTODOS}

Foram realizados dois experimentos no Laboratório de Piscicultura Marinha da Universidade Federal de Santa Catarina. O experimento 1, com 15 dias de duração, foi conduzido entre os dias 14 e 28 de novembro de 2007. Neste experimento utilizou-se larvas recém eclodidas alimentadas exclusivamente com rotíferos Brachionus rotundiformis. No experimento 2, realizado entre os dias 15 de dezembro de 2007 á 15 de janeiro de 2008, utilizou-se larvas de 31 a 62 dias, alimentadas com metanáuplios de Artemia franciscana e com ração comercial (NRD, INVE Aquaculture, Bélgica). Ambos tinham 3 tratamentos, com 12,18 e 24 horas de luz por dia, em triplicata.

\section{MONTAGEM DAS INSTALAÇÕES DE CULTIVO}

Desenvolveu-se uma estrutura de cultivo utilizado para os dois experimentos, com unidades experimentais montadas com vasos plásticos pretos (Nutriplan №4, Nutriplan, Santa Fé do Sul, Brasil) com 4,5 litros de volume, $(18 \mathrm{~cm}$ de altura, $55 \mathrm{~cm}$ de diâmetro inferior e $70 \mathrm{~cm}$ de diâmetro superior). Um mecanismo de drenagem foi acoplado em cada vaso para facilitar a renovação de água. Cada um foi provido de leve aeração, e os fundos foram revestidos por um laminado de PVC para impermeabilização com 1,0 mm de espessura e cor preta (Vinimanta, Sansuy, Embu, Brasil) colados com silicone atóxico Siltrade (Siltrade, Várzea Paulista, Brasil). As unidades experimentais foram acopladas em grades de madeira e dispostas na superfície de um tanque retangular de fibra de vidro com 2,48 x 0,82 x 0,70m, ( 1500 litros de volume). Desse modo, o terço inferior de todas as unidades ficou submerso, possibilitando um ambiente bem homogêneo em temperatura. Termostatos e aquecedores foram responsáveis pela manutenção da temperatura em $\sim 26{ }^{\circ} \mathrm{C}$. Um tanque de água suspenso, independente do tanque principal onde as unidades foram dispostas, serviu de reservatório de água para renovação de cada unidade experimental, com auxílio de micro-mangueiras, por gotejamento lento. A iluminação foi composta por duas lâmpadas fluorescentes de $40 \mathrm{~W}$, posicionadas a $55 \mathrm{~cm}$ de altura em relação ao espelho d’água, com intensidade luminosa média de 1800 lux, registrada com uso de luxímetro digital (Digital lluminator Meter DX-200, INS).

As triplicatas do tratamento com $24 \mathrm{~h}$ de luz foram revestidas com uma lona plástica preta, num extremo do tanque retangular, de modo que à noite sua iluminação contínua não interferisse nos demais tratamentos. No outro extremo, agruparam-se as repetições dos tratamentos $12 \mathrm{~h}$ e $18 \mathrm{~h}$. No tratamento com $12 \mathrm{~h}$ o fotoperíodo foi controlado manualmente, com auxílio de tampas que eram removidas e recolocadas. No caso de $18 \mathrm{~h}$ utilizou-se um "timer" que acionava a luz às 08:00 h e desligava às 02:00 h.

\section{Experimento 1}

\section{MATERIAL BIOLÓGICO}

Utilizaram-se ovos de robalo-peva originados de indução hormonal à desova de reprodutores do plantel do laboratório, segundo método descrito por Cerqueira (2004). Os ovos foram transferidos da incubadora para um béquer com 1 litro de água 
marinha. Com uma pipeta de Bogorov, efetuou-se uma contagem $\mathrm{e}$ depois foram colocados nas unidades experimentais. A densidade era de $190 \pm$ 7,97 ovos por unidade experimental. A taxa de eclosão foi de $95 \%$, resultando em 180 larvas em cada unidade. A média de comprimento padrão ( \pm desvio padrão) de uma amostra de 30 larvas recémeclodidas foi de $2,45 \mathrm{~mm}( \pm 0,05)$.

\section{CULTIVO E FORNECIMENTO DE ALIMENTO}

Rotíferos, Brachionus rotundiformis, foram fornecidos como alimento vivo para larvas a partir do $3^{\circ}$ dia, às 8:00 h e 17:00 h, na proporção de 5 indivíduos $/ \mathrm{mL}$. Os Rotíferos foram alimentados com microalga Nannochloropsis oculata e emulsão comercial (Selco, INVE Aquaculture, Bélgica), fornecida 6 horas antes da colheita. Ao final de cada ciclo de cultivo de 3 dias, iniciava-se um novo, conduzido num tanque cilíndro-cônico de $30 \mathrm{~L}$, provido de aeração e mantido a $28{ }^{\circ} \mathrm{C}$ com salinidade de 25 .

\section{Experimento 2}

\section{MATERIAL BIOLÓGICO}

O experimento teve duração de 32 dias. Larvas previamente cultivadas em tanques de $6000 \mathrm{~L}$, a 26 C, fotoperíodo natural, e alimentadas com Artemia franciscana duas vezes ao dia, foram transferidas, em duas etapas, para as unidades experimentais, na densidade de 35 larvas por tanque (7,7/L). Os valores de comprimento total $(\mathrm{Ct})$ e comprimento padrão $(\mathrm{Cp})$ expressos pela média \pm desvio padrão, de uma mostra de 30 larvas de 31 dias, foram de $8,55 \mathrm{~mm}( \pm 0,94) \mathrm{e}$ $6,95 \mathrm{~mm}( \pm 0,73)$, respectivamente.

\section{CULTIVO E FORNECIMENTO DE ALIMENTO}

Metanáuplios de Artemia franciscana foram cultivados em tanque cilíndrico-cônico de $30 \mathrm{~L}$, mantidos a $28 \stackrel{\circ}{\circ} \mathrm{C}$ e salinidade 35 , enriquecidos com emulsão comercial (Selco, INVE Aquaculture, Bélgica).

Os metanáuplios foram fornecidos numa proporção crescente até 0 início do desmame (transição para o alimento inerte) e decrescente até o seu final. As quantidades foram: $1^{\circ}$ dia: 65/larva; $2^{\circ}$ dia: 102/larva (ambos em duas porções). Do $3^{\circ}$ ao $5^{\circ}$ dia: 130/larva; no $6^{\circ}$ dia: 195/larva (ambos em 3 porções). Do $7^{\circ}$ ao $8^{\circ}$ dia: 4 porções de 195 metanáuplios por larva. Do $9 \stackrel{\circ}{\circ}$ ao $11^{\circ}$ dia, quando se iniciou o processo de desmame, redução de 50\% (97,5 indivíduos por larva), 4 vezes ao dia. No $12^{\circ}$ e $13^{\circ}$ dias a freqüência foi de 3 vezes. Do $14^{\circ}$ ao $17^{\circ}$ dia 2 vezes, e no $19^{\circ}$ dia apenas ração. $O$ processo de desmame durou 11 dias (dos 9 aos 19 dias). O fornecimento de ração foi aumentando em função do consumo.

No período de 49 a 60 dias de idade, 0 tratamento $12 \mathrm{~h}$ foi alimentado 4 vezes por dia com ração, nos horários 08:00 h, 11:00 h, 14:00 h e 17:00 h. $\mathrm{O}$ tratamento $18 \mathrm{~h} 6$ vezes, nos horários 08:00 h, 11:00 h, 14:00 h, 17:00 h 20:00 h e 23:00 h. E o tratamento $24 \mathrm{~h} 8$ vezes, nos horários 8:00 h, 11:00 h, 14:00 h, 17:00 h, 20:00 h, 23:00 h, 02:00 h e 05:00 h. A partir de 23:00 $\mathrm{h}$ e até 05:00 $\mathrm{h}$ foi utilizado um alimentador automático e nos demais horários a alimentação foi manual. Duas renovações de água foram realizadas por dia, durante uma hora, ocasião em que também se sifonavam os restos de ração. A taxa de renovação da água foi de 10 a $30 \%$ no experimento 1, iniciada após a primeira metade do experimento, e de 30 a $100 \%$ no experimento 2. A água utilizada foi esterilizada por UV, constantemente aerada e mantida a $26{ }^{\circ} \mathrm{C}$ com aquecedores elétricos.

\section{PARÂMETROS AVALIADOS}

No Experimento 1, a salinidade e a temperatura de cada unidade experimental foram monitoradas diariamente às 8:00 h. A amônia total foi medida no meio e ao final do experimento e se manteve inferior a $0,25 \mathrm{mg} / \mathrm{L}$. A salinidade variou de 35,3 a 35,8 e a temperatura de 25,1 a $25,5^{\circ} \mathrm{C}$. Os níveis de oxigênio dissolvido ficaram entre 5 e $6 \mathrm{mg} / \mathrm{L}$. Ao final do experimento, avaliou-se a sobrevivência (\%) e o comprimento padrão $(\mathrm{mm})$. Os dados são apresentados como média \pm desvio padrão.

No experimento 2, a salinidade variou de 33,8 a 33,9 e a temperatura de 26,2 a $26,4{ }^{\circ} \mathrm{C}$. Os níveis de oxigênio dissolvido entre 5 e $6 \mathrm{mg} / \mathrm{L}$.

Compararam-se sobrevivência (\%), comprimento total $(\mathrm{cm})$ e peso $(\mathrm{g})$. Com os dados obtidos, calculou-se a Taxa de Crescimento Específico Diário: TCE $=[(\mathrm{In} \mathrm{pf}-$ In pi) / t] x 100; onde pf é o peso final (g), pi é o peso 
inicial (g) e t é o tempo em dias (Houde 1989 apud Okamoto 2006); o fator de condição de Fulton (K= peso/comprimento padrão ${ }^{3}$ x 100); o Índice de Conversão Alimentar (ICA): consumo de ração + Artemia (g)/ ganho de peso (g) aos 31 dias; a Taxa de Alimentação: consumo/ biomassa na despesca x 100 .

Para o tratamento estatístico, os valores de sobrevivência foram convertidos para arco-seno. Para os dois experimentos foi feita uma Análise de Variância simples, ao nível de significância de 5\%. Quando foram encontradas diferenças significativas, foi aplicado o Teste de Tukey. Os dados são apresentados como média \pm desvio padrão.

\section{RESULTADOS}

\section{Experimento 1}

Para sobrevivência encontrou-se os valores de $3,14 \%$ ( $\pm 6,09), 9,25 \%( \pm 10,09)$ e $4,62 \%( \pm 1,15)$ para os tratamentos $12 \mathrm{~h}, 18 \mathrm{~h}$ e $24 \mathrm{~h}$ respectivamente. Porém, não diferiram significativamente (Figura 1).

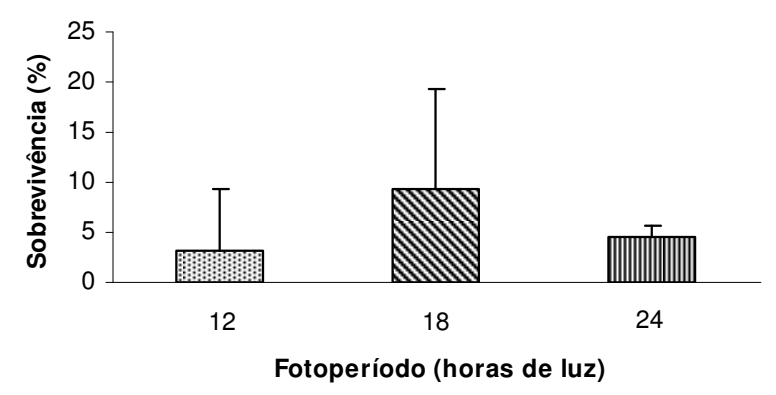

FIGURA 1 - Sobrevivência (média \pm dp) de larvas de robalo-peva de 14 dias, submetidas a fotoperíodos de 12, 18 e 24 horas.

Para comprimento padrão, o melhor resultado obtido ocorreu no tratamento $24 \mathrm{~h}$, de $3,70 \mathrm{~mm}( \pm 0,1)$, seguido pelo tratamento $18 \mathrm{~h}$ e $12 \mathrm{~h}$, com $3,49 \mathrm{~mm}( \pm$ $0,22)$ e $3,35 \mathrm{~mm}( \pm 0,15)$ respectivamente (Figura 2).

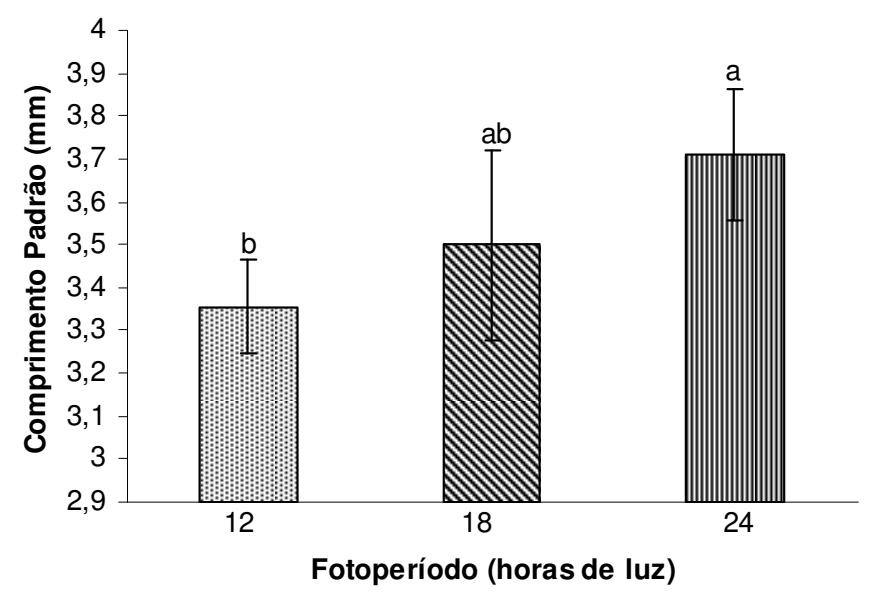

FIGURA 2 - Comprimento padrão (média \pm dp) em larvas de robalo-peva de 14 dias submetidas a fotoperíodos de 12,18 e 24 horas. Letras diferentes indicam diferenças significativas.

\section{Experimento 2}

$\mathrm{Na}$ tabela 1 são apresentados os valores dos parâmetros avaliados a partir dos registros de alimentação e desempenho em crescimento das larvas ao final do segundo experimento. 
TABELA 1 - Índice de conversão alimentar (ICA), Taxa de alimentação, Fator de Condição de Fulton (K) e Taxa de Crescimento Específico (TCE) para larvas de robalo-peva com 61 dias. de idade. Os valores representam as médias $\pm \mathrm{dp}$. Letras diferentes representam diferenças significativas. ANOVA; Tukey.

\begin{tabular}{ccccc}
\hline Tratamentos & ICA & Tx. Alim. (\%) & K & TCE (\%) \\
\hline $\mathbf{1 2} \mathbf{h}$ & $1,510 \pm 0,07 \mathrm{a}$ & $8,94 \pm 2,54 \mathrm{ab}$ & $1,92 \pm 0,05 \mathrm{a}$ & $8,99 \pm 0,73 \mathrm{a}$ \\
& & & & \\
\hline $\mathbf{1 8} \mathbf{h}$ & $0,962 \pm 0,26 \mathrm{~b}$ & $7,36 \pm 1,97 \mathrm{a}$ & $2,27 \pm 0,69 \mathrm{a}$ & $10,2 \pm 0,71 \mathrm{a}$ \\
\hline $\mathbf{2 4} \mathbf{h}$ & $1,661 \pm 0,16 \mathrm{a}$ & $13,93 \pm 1,25 \mathrm{~b}$ & $1,89 \pm 0,17 \mathrm{a}$ & $10,6 \pm 0,57 \mathrm{a}$ \\
\hline
\end{tabular}

Para sobrevivência, cujos valores foram de $91,42 \%$ ( $\pm 7,56), 87,42 \%( \pm 5,94)$ e $77,14 \%( \pm 7,56)$ para os tratamentos $12 \mathrm{~h}, 18 \mathrm{~h}$ e $24 \mathrm{~h}$, respectivamente, não foram encontradas diferenças significativas (Figura 3).

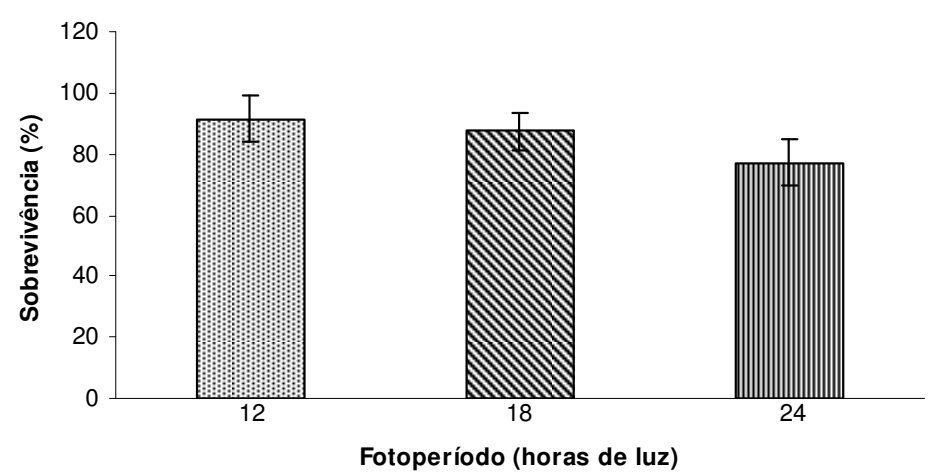

FIGURA 3 - Sobrevivência (média \pm dp) de larvas de robalo-peva de 62 dias submetidas a fotoperíodos de 12, 18 e 24 horas.

Nos valores de peso $(\mathrm{g})$ ocorreram diferenças significativas entre todos os tratamentos. O melhor resultado foi encontrado para 0 tratamento $18 \mathrm{~h}$
$(0,1858 \mathrm{~g} \pm 0,04)$, seguido do tratamento $24 \mathrm{~h}(0,1595$ $\mathrm{g} \pm 0,02)$ e do tratamento $12 \mathrm{~h}(0,1119 \mathrm{~g} \pm 0,02)$. (Figura 4).

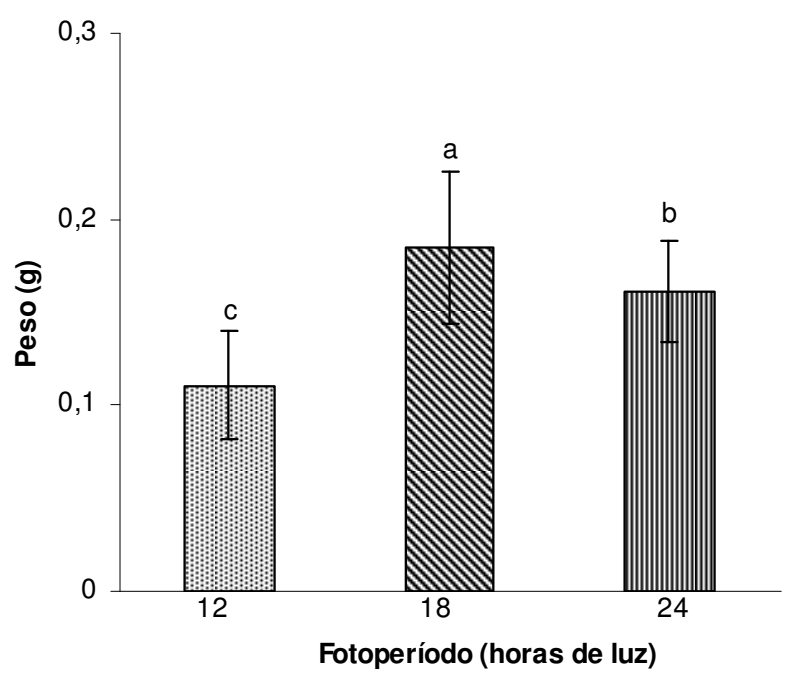

FIGURA 4 - Peso (média \pm dp) de larvas de robalo-peva de 62 dias submetidas a fotoperíodos de 12, 18 e 24 horas. Letras diferentes indicam diferenças significativas 
Para comprimento total $(\mathrm{cm})$, os valores foram significativamente maiores no tratamento $18 \mathrm{~h}$, resultando em larvas de $2,60 \mathrm{~cm} \pm 0,13$, seguido dos tratamentos $24 \mathrm{~h}$ e $12 \mathrm{~h}$, cujos valores diferiram significativamente, sendo de $2,42 \mathrm{~cm} \pm 0,18$ para 0 tratamento $24 \mathrm{~h}$ e $2,19 \mathrm{~cm} \pm 0,21$ para o tratamento $24 \mathrm{~h}$ (Figura 5).

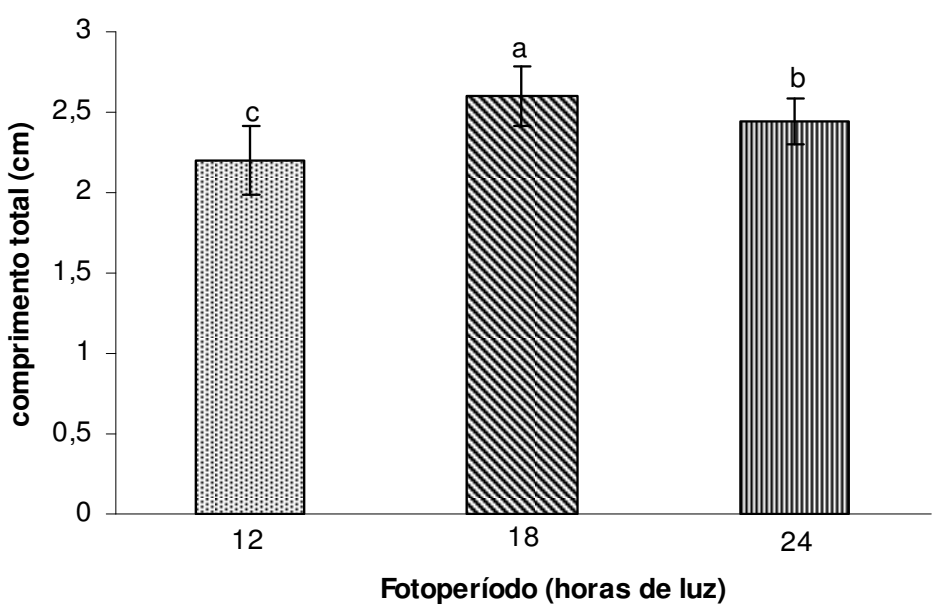

FIGURA 5 - Comprimento total (média \pm dp) em larvas de robalo-peva de 62 dias submetidas a fotoperíodos de 12,18 e 24 horas. Letras diferentes indicam diferenças significativas.

\section{DISCUSSÃO}

\section{Experimento 1}

OS resultados médios de sobrevivência estão dentro da faixa normalmente observada para a espécie. A mortalidade ocorrida está provavelmente associada ao período de transição entre a alimentação endógena e exógena, ocorrida entre os dias 4 e 11 de idade. Nesta idade as larvas são bastante frágeis (Alvarez-Lajonchère et al. 2002a). Em trabalhos prévios com o rabalo-peva, as sobrevivências iniciais obtidas foram variadas, dependendo do tratamento aplicado e também da origem das larvas. Por exemplo, Cerqueira e Brugger (2001), avaliando a influência da intensidade luminosa, observaram sobrevivências entre $0,06 \%$ e $16 \%$. Seifert (1996), em trabalho que testava diferentes fontes de alimento vivo na primeira alimentação, obteve sobrevivências de $0,2 \%$ a 2,4\%. Em um experimento comparando a alimentação de larvas de robalo com rotíferos enriquecidos com microalgas, fermento ou emulsões comerciais, as taxas de sobrevivência foram de 1,8\% a 2,7\% (Seifert et al., 2001). Por outro lado, maiores taxas de sobrevivência foram obtidas por Araújo et al. (2000), que, submetendo larvas a diferentes salinidades, tiveram entre $13,8 \%$ e $23,5 \%$ de sobrevivência. Temple et al. (2004), investigando diferentes densidades de rotíferos, obtiveram até $38,8 \%$ de sobrevivência. Esta disparidade de resultados poderia ser explicada, não somente com os fatores estudados individualmente em diversos experimentos, mas também pela origem das larvas, considerando o método de indução à desova e a qualidade do plantel de reprodutores cultivados (Cerqueira e Tsuzuki, 2009).

Portanto, podemos considerar que as larvas não sofreram influência negativa dos tratamentos experimentais, uma vez que os valores de sobrevivência observados estão dentro da margem esperada para a espécie, neste estágio de desenvolvimento.

Os valores obtidos para comprimento padrão, que variou de $3,35 \mathrm{~mm}$ a $3,70 \mathrm{~mm}$, foram semelhantes aos valores obtidos por AlvarezLajonchère et al. (2002a) em larvas de mesma idade.

Em longos períodos de disponibilidade visual das presas, geralmente se tem um maior crescimento de larvas de peixes marinhos (Howell et al., 1998 apud Cañavate, 2006). Diversas espécies são descritas apresentando melhor crescimento sob fotoperíodos longos, como, por exemplo: Sparus aurata (Tandler e Helps, 1985), Siganus guttatus (Duray e Kohno, 1988), Lates calcarifer (Barlow et al.,1995), Pagrus auratus (Fielder et al., 2002), Gadus morhua (Puvanendram e Brown, 2002) e Latris lineata (Trotter et al., 2003). 
Porém, também existem espécies em que o crescimento não foi favorecido com o aumento do fotoperíodo, como descrito para Melanogramus aeglefinus (Downing e Litvak, 1999) e Morone saxalitis (Martim-Robichaud e Peterson, 1998). Larvas de Dentex dentex também apresentaram melhor crescimento no fotoperíodo de $18 \mathrm{~h}$ do que sob iluminação permanente (Abellan et al., 2000 apud Cañavate, 2006). A extensão do fotoperíodo natural para uma iluminação contínua também não resultou em melhora do crescimento de larvas de Solea senegalensis (Cañavate, op.cit). Por outro lado, em larvas de robalo europeu $D$. labrax mantidas na escuridão total até a exaustão da reserva vitelina, verificou-se inibição do comportamento forrageiro, poupando-as de um gasto de energia desnecessário (Chatain, 1991 apud Bromage, 1994). Para robalopeva, o tratamento $18 \mathrm{~h}$ seria o mais indicado para cultivo larval neste estágio de desenvolvimento, por beneficiar o desempenho em crescimento e não representar desvantagem na sobrevivência em relação ao regime de iluminação contínua.

\section{Experimento 2}

A biometria inicial, com larvas de 30 dias, revelou valores semelhantes de comprimento total descritos para a espécie nesta mesma idade. AlvarezLajonchère et al. (2002a) encontraram, para larvas de robalo-peva de 30 dias, comprimento total de $9,64 \mathrm{~mm}$ $\pm 1,02$, ligeiramente superiores aos obtidos no presente trabalho. Em ambos os estudos o fotoperíodo era o natural. No entanto, quando comparamos os valores do crescimento ao final do experimento, com 62 dias de idade, com larvas de mesma idade cultivadas sob protocolos de produção em grande escala, observamos valores inferiores. Esta diferença pode ter sido causada pela utilização de pequenas unidades experimentais. Por exemplo, para larvas de 68 dias de idade, cultivadas em tanques de $4000 \mathrm{~L}$, Alvarez-Lajonchère et al. (2002b) observaram valores de 3,6 $\pm 0,06 \mathrm{~cm}$ a $4,5 \pm 0,06 \mathrm{~cm}$ de comprimento total, superiores ao maior valor de comprimento total encontrado para larvas no presente trabalho, que variou de $2,2 \mathrm{~cm} \pm 0,21$ a $2,59 \mathrm{~cm} \pm 0,13$. No primeiro experimento, a unidade experimental pareceu não limitar o crescimento das larvas nos primeiros 15 dias.
As larvas com 31 dias de idade encontravam-se em início de processo de metamorfose, o que poderia explicar a mortalidade ocorrida nesta ocasião, além do processo de desmame em curso. Alvarez-Lajonchère et al. (2002a), relataram que o segundo momento crítico do desenvolvimento larval de robalo-peva é justamente por volta dos 30 dias de idade. Entretanto, as mortalidades foram bem menores quando comparadas com as que ocorreram no primeiro experimento, pois a visão e a capacidade de alimentação já estavam plenamente desenvolvidas. Em todo caso, não houve diferenças significativas entre os tratamentos com relação à sobrevivência.

Quanto ao peso, o tratamento $18 \mathrm{~h}$ foi significativamente superior aos tratamentos $12 \mathrm{~h}$ e 24 $\mathrm{h}$, apesar de, no terço final do experimento, o tratamento $24 \mathrm{~h}$ ter recebido quatro doses a mais de ração que o tratamento $12 \mathrm{~h}$ e o tratamento $18 \mathrm{~h}$ ter recebido apenas duas a mais. Isso nos sugere que a maior disponibilidade de tempo para alimentação, propiciada pela iluminação e consequentemente a visualização do alimento, acompanhada por mais fornecimento de ração, como ocorreu entre os tratamentos $24 \mathrm{~h}$ e $18 \mathrm{~h}$, não foi necessariamente convertida em maior crescimento. Da mesma forma, o Índice de Conversão Alimentar (ICA) obtido com o tratamento $18 \mathrm{~h}$ foi mais eficiente. De modo semelhante, analisando-se o crescimento, alguns autores sugeriram que, apesar do aumento do fotoperíodo prover uma melhora em decorrência do maior comportamento forrageiro (Kiyono e Hirano, 1981; Tandler e Helps, 1985, Duray e Kohno, 1988; Hart et al., 1996 apud Trotter 2003), também não houve diferença significativa entre os tratamentos 24 $\mathrm{h}$ e $18 \mathrm{~h}$, sugerindo haver um limite para que esta vantagem seja verificada. Quando este limite é ultrapassado, não há mais efeitos positivos no crescimento. Barlow et al. (1995) observaram que juvenis de robalo asiático Lates calcarifer, sob iluminação por 24 horas, tiveram um aumento de $40 \%$ no consumo de alimento quando comparado com o fotoperíodo de $12 \mathrm{~h}$. Porém, a quantia extra de alimento foi gasta com energia "não produtiva" associada ao aumento da atividade dos peixes. Isto pode ser atribuído à influência dos ritmos circadianos na alimentação e no metabolismo (Cerqueira e Chatain, 1991; Boujard e Leatherland, 1992). Sob a 
influência de tais ritmos, a captura da presa e a digestão poderiam ser limitadas (Cerqueira e Chatain, 1991). As larvas poderiam continuar nadando ativamente, gastando até 2,5 vezes mais energia do que se estivessem no escuro (Laurence, 1977 apud Trotter, 2003). Dowd e Houde (1980 apud Cerqueira e Chatain, 1991) sugerem que a quantidade de alimento que as larvas podem ingerir por dia é limitada, e que indivíduos cultivados sob longos fotoperíodos podem gastar mais energia do que 0 consumo de alimento pode propiciar.

O maior peso e comprimento total obtidos nos tratamentos $18 \mathrm{~h}$ e $24 \mathrm{~h}$ em relação ao tratamento 12 h influenciaram no Fator de Condição, porém não houve diferenças significativas entre os tratamentos. A taxa de crescimento específico, apesar da aparente diferença numérica entre os tratamentos, não houve diferenças estatísticas.

Os valores obtidos para índice de conversão alimentar (ICA) e taxa de crescimento específico não diferiram muito de trabalhos anteriores com a mesma espécie. Alvarez-Lajonchère et al. (2002b) obtiveram, para larvas de $C$. parallelus cultivadas por 88 dias, taxa de conversão alimentar de 1,17 e taxa de crescimento específico diário em peso, de 13\%, considerando somente o período pós-desmame. Em outra ocasião, considerando larvas de robalo-peva no período pós-desmame de 66 a 90 dias de idade, encontraram taxa de conversão alimentar de 1,28 e taxa de crescimento específico diário de 8,5\% (Alvarez-Lajonchère et al., 2004).

\section{CONCLUSÃO}

Podemos sugerir um regime de iluminação composto por 18 horas de luz seguidas por 6 horas de escuro, por apresentar melhor relação iluminação $x$ desempenho, nos dois períodos larvais estudados. É possível que isto também ocorrerá quando o mesmo fotoperíodo for adotado no protocolo de produção em escala comercial. Entretanto, ainda há necessidade de novos trabalhos que avaliem a relação do fotoperiodo com o crescimento e a sobrevivência de larvas em diferentes condições de cultivo, como o tamanho dos tanques, mas também considerando todas as fases do desenvolvimento larval.

\section{AGRADECIMENTOS E FINANCIAMENTO}

Os autores agradecem ao Conselho Nacional de Desenvolvimento Cientifico e Tecnológico (CNPQ), pelo financiamento concedido para a realização deste trabalho.

\section{REFERÊNCIAS BIBLIOGRÁFICAS}

ALVAREZ-LAJONCHÈRE, L. et al. First basis for a sustained juvenile production technology of fat snook Centropomus parallelus Poey. Hidrobiologica v.14, n.1, p.37-45, 2004.

ALVAREZ-LAJONCHÈRE, L. et al. Desarrollo embrionario y primeros estadios larvales del robalo gordo, Centropomus parallelus Poey (Pisces, Centropomidae) con interés para su cultivo. Hidrobiologica, v.12, n.2, p.89-99, 2002a.

ALVAREZ-LAJONCHĖRE, L.. et al. Mass production of juveniles of the snook Centropomus parallelus in Brazil. J.Word Aquacult. Soc. v.33, n.4, p.506-516, 2002b.

BARLOW, C.G. et al. Effects of photoperiod on growth, survival and feeding periodicity of larval and juvenile barramundi Lates calcarifer (Bloch). Aquaculture, Amsterdam, v.138, p.159- 168, 1995.

BOEUF, G.; LE BAIL, P.Y. Does Light have an influence on fish growth? Aquaculture, Amsterdam, v.177, p.129-152, 1999.

BOUJARD, T.; LEATHERLAND, J.F. Circadian rhythms and feeding time in fishes. Environ. Biol. Fishes V.35 (2), P.109- 131, 1992.

BRENNAN, N.P. et al. Predator-free enclousures improve postrelease survival os stocked common snook. J exp Mar Biol Ecol. n. 335, p. 302-311, 2006

BROMAGE, N.R.; ROBERTS, R.J. Editors, Broodstock Management and Egg and Larval Quality, Blackwell, Oxford, (1994), $432 \mathrm{p}$.

CAÑAVATE, J.P. et al. Feeding and development of Senegal sole (Solea senegalensis) larvae reared in different photoperiods. Aquaculture, Amsterdam, v.258, p.368-377, 2006.

CERQUEIRA, V. R. Cultivo de Peixes Marinhos. In: POLI, C.R. et al. (Ed.). Aquicultura: Experiências Brasileiras. Florianópolis: Multitarefa Editora, 2004. cap XV. p. 369-406.

CERQUEIRA, V. R. Cultivo do robalo. Aspectos da reprodução, larvicultura e engorda. Florianópolis: UFSC. Edição do autor, 2002.

CERQUEIRA, V.R.; BRÜGGER, A.M. Effect of Light Intensity on Initial Survival of Fat Snook (Centropomus parallelus, Pisces: Centropomidae) Larvae. Braz Arch Biol Technol v. 44, n.4, p. 343-349, 2001.

CERQUEIRA, V.R.; CHATAIN, B. Photoperiodic effects on the growth and feeding rhythm of European seabass, Dicentrarchus labrax, larvae in intensive rearing. In: Lavens, P., Sorgeloos, P., Jasper, E., Ollevier, F. (Eds.), Larvi '91-Fish and Crustacean Larviculture Symposium, Gent, Belgium. Spec. Publ. Eur. Aquaculture. Soc. European Aquaculture Society, Special Publication 15, Ghent, vol. 15, p. 304-306, 1991.

CERQUEIRA, V.R.; TSUZUKI, M.Y. A review of spawning induction, larviculture, and juvenile rearing of the fat snook, Centropomus parallelus. Fish Physiol Biochem, Springer, 2009.

DOWNING, G.; LITVAK, M.K. The effect of photoperiod, tank colour and light intensity on growth of larval haddock. Aquac. Int.v. 7, p. 369-382, 1999. 
DURAY, M.; KOHNO, H. Effects of continuous lighting on growth and survival of first-feeding larval rabbitfish, Siganus guttatus. Aquaculture, Amsterdam, v.109, p. 311-32 I, 1988.

EFTHIMIOU, S. et al. Growth, food conversion and agonistic behaviour in common dentex (Dentex dentex) juveniles fed on pelleted moist and dry diets. Aquatic Living Resources, Paris, v.7, p.267-275, 1994.

FAO 2006: Capture production 2004. FAO yearbook. Fishery statistics. Capture production. v. 98/1. Rome, 2006.

FIELDER, D.S. et al. Effect of photoperiod on growth and survival of snapper Pagrus auratus larvae. Aquaculture, Amsterdam, v. 211, p.135-150, 2002.

HART, P.R. et al. Effects of photoperiod, temperature and salinity on hatchery-reared larvae of the greenback flounder (Rhombosolea tapirina Gunther, 1862). Aquaculture, Amsterdam, v.144, p.303-311, 1996.

MARTIN-ROBICHAUD, D.J.; PETERSON, R.H. Effects of light intensity, tank colour and photoperiod on swimbladder inflation success in larval stripped bass, Morone saxatilis (Walbaum). Aquac. Res. v. 29, p.539-547, 1998.

OKAMOTO, M.H. et al. Efeito da temperatura sobre o cresimento e a sobrevivência de juvenis da tainha Mugil platanus (Ghünter, 1980). Atlantica, Rio Grande, v.28, n.1. p.61-66, 2006.

PUVANENDRAM, V.; BROWN, J.A. Foraging, growth and survival of Atlantic cod larvae reared in different light intensities and photoperiods. Aquaculture, Amsterdam, v. 214, p.131-151, 2002.

RIVAS, L.R. Systematic review of the perciform fishes of the genus Centropomus. Copeia, p. 579-611, 1986.

SEIFFERT, M.B. Influência do alimento vivo e do regime alimentar na larvicultura do robalo Centropomus parallelus (Poey, 1860). Dissertação de mestrado, Universidade Federal de Santa Catarina, 1996.

SEIFFERT, M.B. et al. Effect of dietary ( $n-3)$ highly unsaturated fatty acids (HUFA) on growth and survival of fat snook (Centropomus parallelus, Pisces: Centropomidae) larvae during first feeding. Braz J Med Biol Res. v.34, n.5, p. 645-651, 2001.

TANDLER, A.; HELPS, S. The effects of photoperiod and water exchange rate on growth and survival of gilthead sea bream (Sparus aurata, Linnaeus; Sparidae) from hatching to metamorphosis in mass rearing systems. Aquaculture, Amsterdam, v. 48, p.71-82, 1985.

TEMPLE, S. et al. The effects of lowering prey density on the growth, survival and foraging behaviour of larval fat snook (Centropomus parallelus poey 1860). Aquaculture, Amsterdam, V.233, p.205-217, 2004.

TROTTER, A.J. et al. Effects of photoperiod and light intensity on initial swim bladder inflation, growth and post-inflation viability in cultured striped trumpeter (Latris lineata) larvae. Aquaculture, Amsterdam, V.224, p.141-158, 2003. 
